\title{
Spin Quantization in a Classical Model
}

\author{
David L. Selke
}

\section{ABSTRACT}

The origins of quantum theory lie in a failure to explain certain phenomena using classical theory. But despite its adoption, quantum theory abounds with concepts described as "weird" or "spooky." Even Feynman said that no one understands quantum theory. We revisit the Stern-Gerlach experiment that gave rise to the concept of spin and its quantization from a classical point of view. We present a simplified model of the classical electron which has two stable states which separate into two beams in the presence of an inhomogenous magnetic field.

Keywords: electron, classical, Stern-Gerlach, spin, GUT-CP.

Published Online: February 6, 2021

ISSN: $2684-4451$

DOI :10.24018/ejphysics.2021.3.1.47

\section{L. Selke*}

New Model Arts, Chandler, AZ, USA. (e-mail: dselke ${ }^{\circledR}$ hotmail.com)

*Corresponding Author

\section{INTRODUCTION}

Mills' Grand Unified Theory of Classical Physics (GUT$\mathrm{CP})$ gives a classical electron model that is a complicated arrangement of current loops that are great circles making up a spherical shell that encloses the nucleus - an atomic orbital [1]. We will show that the essential behavior of the Stern-Gerlach experiment is already present with just two current loops. We assume that the system of two current loops is rigid, so that the total force is just the vector sum of the individual forces.

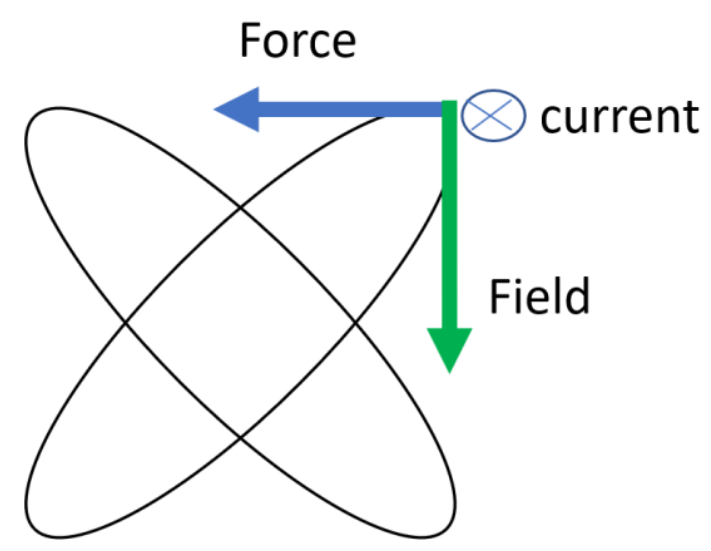

Fig. 1. With a (conventional) current vector shown into the page at the top right corner, and a field directed down, the resulting Lorentz force is to the left (blue arrow), according to the right hand rule for the cross product.

\section{SIMPLIFIED ELECTRON MODEL}

Our model, consisting of two orthogonal current loops, is shown in Figure 1. Nearby points on the current loop have similar forces. We will explicitly show the currents at the four corners, but the forces at other points are perpendicular to the current and field, so they point toward the vertical axis. These other points' into the page and out of the page components of force can be seen to cancel on a point-pairwise basis as one point traverses the loop clockwise and the other counterclockwise starting at a corner. After this cancellation the remaining components behave as shown, just with a smaller magnitude.

\section{UnSTABLE STATES}

Most of the states of rotational position feel a net torque, as shown in Fig. 2. These states will rotate until a stable state of rotational position is reached. Notice that the torque and subsequent alignment will occur in a uniform magnetic field as well as an inhomogenous one.
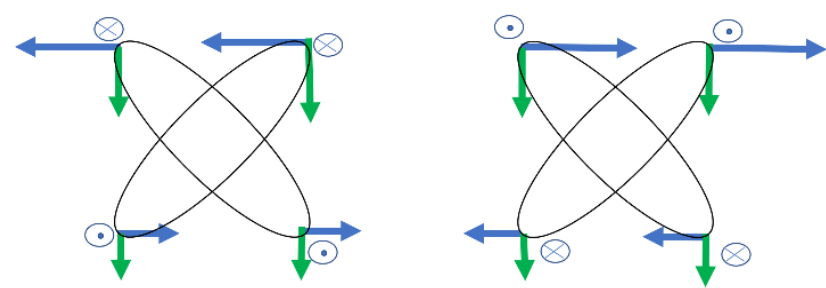

Fig. 2. The two states shown experience a net torque due to the Lorentz force. Smaller vectors indicate weaker field and force.

\section{STABLE STATES}

Two states experience zero net torque. They are shown in Fig. 3.
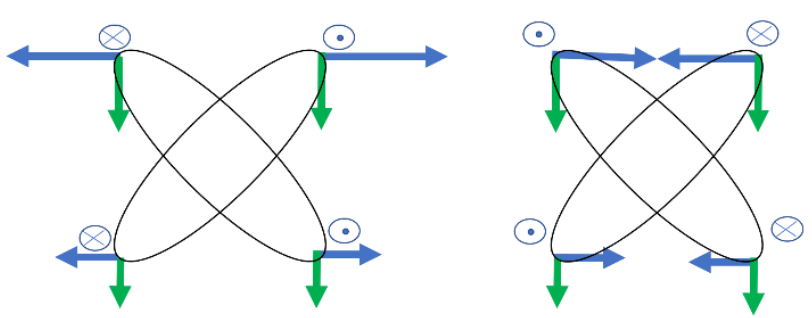

Fig. 3. The two states shown have no net torque due to the Lorentz force. 


\section{Net Force on Stable States}

The two stable states' force vectors may be resolved into a component perpendicular to the radius and a component parallel to the radius as in Fig. 4. The perpendicular or normal component gives rise to a torque, however due to symmetry the torques are in balance. The parallel or radial components at the top of the electron are stronger than those at the bottom because of the field gradient in the SternGerlach experiment. This causes a net force up on the left electron, and a net force down on the right electron. This causes the separation of beams. However note that with a uniform field, the top and bottom forces would be in balance and there would be no separation of beams.
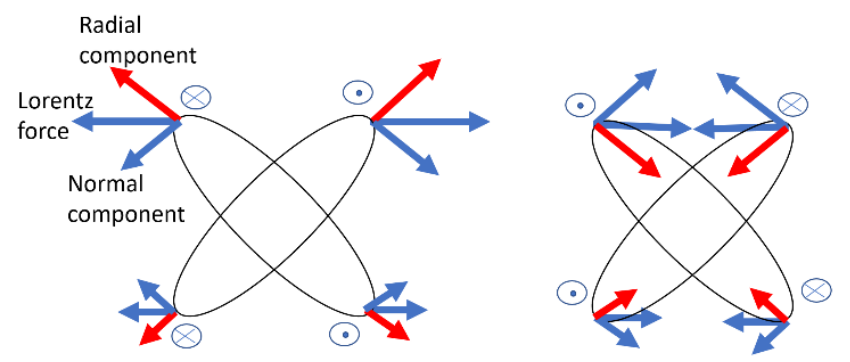

Fig. 4. The radial vectors (red) contribute to a net force up or down on the two stable states.

\section{CONCLUSION}

A simplified model inspired by Mills' atomic orbital was shown to exhibit the behavior of the Stern-Gerlach experiment, including: two stable states, two separated beams in an inhomogenous field, and one beam in a homogenous field.

\section{REFERENCES}

[1] R. L. Mills, The Grand Unified Theory of Classical Physics. Brilliant Light Power, Cranbury, NJ: Retrieved from https://www.brilliantlightpower.com/book-download-and-streaming/. 